\title{
Liposomal HPV-16 E6/E7 Multipeptide Vaccine PDS0101
}

National Cancer Institute

\section{Source}

National Cancer Institute. Liposomal HPV-16 E6/E7 Multipeptide Vaccine PDS0101. NCI

Thesaurus. Code C111688.

A liposomal nanoparticle-based therapeutic vaccine composed of the cationic lipid RDOTAP (R-enantiomer of 1,2-dioleoyl-3-trimethylammonium-propane chloride) encapsulating six human papillomavirus 16 (HPV-16) E6 and E7 peptides, with potential immunostimulating activity. Upon subcutaneous administration of the liposomal HPV-16 E6 and E7 multipeptide vaccine, the nanoparticles are taken up by antigen presenting cells (APCs), specifically dendritic cells (DCs), which may stimulate the immune system to induce a cytotoxic T-lymphocyte response (CTL) against HPV-16 E6 and E7-expressing tumor cells. HPV-16 E6 and E7 are oncoproteins that play a key role in the tumorigenesis of a variety of cancers. 